\title{
Asymptotic stability and disturbance attenuation properties for a class of networked control systems
}

\author{
Hai LIN ${ }^{1}$, Guisheng ZHAI ${ }^{2}$, Panos J. ANTSAKLIS ${ }^{1}$, \\ (1. Deparngent of Elecirical Engincering, University of Notre Dame. Notre Dame IN 46556. USA: \\ 2. Department of Mechanical Engineering. Osaka Prelecture Universily. Sakaj Osaki 599-8531. Japan)
}

\begin{abstract}
In this paper, stability and disturbance atteruation issues for a class of Networked Control Systems (NCSs) under uncertain access delay and packet dropout effects are considered. Our aim is to tind conditions on the delay and packet dropout rate, under which the system stability and $\mathrm{H}_{x}$ disturbance attenuation properties are prescrved to a desired level. The basic idea in this paper is to formulate such Networked Control System as a discrete-time switched system. Then the NCSs" stability and performance problems can be reduced to the corresponding problems for switched systems, which have been studied for decades and for which a number of results are available in the literature. The techniques in this paper are based on recent progress in the discrete-time switched systems and piecewise Lyapunov functions.

Keywords: Networked control systems; Switched systems; Piecewise quadratic Lyapunov function
\end{abstract}

\section{Introduction}

By Networked Control Systems (NCSs), we mean feedback control systems where networks, typically digital band-limited serial communication channels, are used for the connections between spatially distributed system components like sensors and actuators to controllers, see Fig. l for illustration. These channels may be shared by other feedback control loops. In traditional feedback control systems, these connections are established by point-to-point cables. Compared with point-to-point cables, the introduction of serial communication networks has several advantages, such as high system testability and resource utilization, as well as low weight, space, power and wiring requirements $[1,2]$. These advantages have made the networks connecting sensors/actuators to controllers increasingly popular in many applications, including traffic control, satellite clusters, mobile robotics, etc. However, the connection by digital serial communication channels also brings several new challenges for our control community. For example, the network link is band-limited, so one needs to quantize the signals and send digital bits. In addition, the network could be noisy and collisions between packets could occur, then one needs to concern the packet dropouts and delays etc. Recently, modeling, analysis and control of networked control systems with limited communication capability has emerged as a topic of significant interest to control community, see for example [ $\sim 5]$, and recent special issue [6].

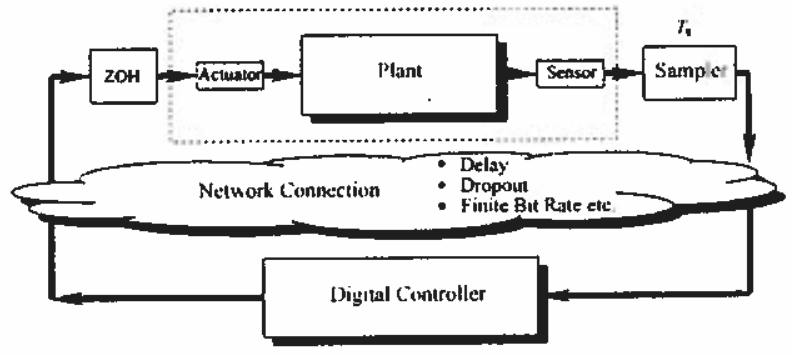

Fig. I The block diagram for a lypical Networked Control Sysilem.

Time delays typically have negative eftects on the NCSs ${ }^{+}$ stability and performance. There are several situations where time delays may arise. First. transmission delays may occur through the data rate limited communication channels. Secondly, channels in NCSs are usually shared by multiple sources of data, and multiplexed by a timedivision method. Therefore, there are delays caused by a node waiting to send out a message through a busy channel. 
which are usually called accessing delays and serve as the main source of delays in NCSs. There are also some delays caused by processing and propagation, which are usually negligible in NCSs. Another interesting problem in NCSs is the packet dropout phenomenon. Because of the uncertainties and noise in the communication channel, there may exist unavoidable errors or losses in the transmitted packet cven when an error control coding and/or Automatic Repeat reQuest ( $A R Q$ ) mechanisms are employed. If this happens, the corrupted packets are dropped and the receivers (controller or actuator) instead use the most recently received packets. Packet dropouts may also occur when one packet. say sampled values from the sensor, reaches the destination later than its successors. In this situation, the old packet is dropped, and its successor packet is used instead. There is another important issue in NCSs, namely the quantization effect. With the finite bit-rate constraints, quantization has to be taken into consideration in NCSs. Therefore, quantization and limited bit rate issues have attracted many researchers' attention with the aim to identify the minimum bit rate required to stabilize a NCS, see for example $[3,5,7 \sim 9]$. In this paper, we will focus on packet exchange networks, in which the minimum unit of data transmission is the packet which typically consists of several hundred bits. Therefore, sending a single bit or several hundred bits do not result in a significant difference in the network resource usage. Hence, we will omit the quantization effects here and focus our attention on the effects of network induced delays and packet dropouts on NCSs' stability and performance.

The effects of network induced delay on the NCS's stability have been studied in the literature. In [ I0]. the delay was assumed to be constant and hence the NCS could be transformed into a time-invariant discrete-lime system. Therefore. the NCS's stability could be checked by verifying whether or not an angmented matrix is a Schur matrix or not. Since inost network protocols introduce delays that can vary from packet to packet, the authors extended the results to non-constant delay case in 12). They employed Lyapunov methods, in particular a common quadratic Lyapunov lunetion. to study bounds on the maximum delay allowed by the NCSs. However. the choice of a common quadratic Lyapunov function could make the conclusion for maximum allowed delay conservative in some cases. The packet dropouts have also been studied, and there are two typical ways to model packet dropouts in the literature. The first approach assumes that the packet dropouts follow certain probability distributions, which is difficult to verify, and describes NCSs with packet dropouts via stochastic models, such as Markovian jump linear systems. The second approach is deterministic, and specifies the dropouts in the time average sense or in terms of bounds on maximum allowed consecutive dropouts. For example, [11] modeled a class of NCSs with package dropouts as asynchronous dynamical systems, and derived a sufficient condition on packet dropouts in the time-average sense for NCSs' stability based on common Lyapunov function approach. Notice that most of the results obtained so far are for the NCS's stability problem, and the delay and packet dropouts are usually dealt with separately.

In this paper, we will consider both network induced delay and packet dropouts in a unified switched system model. In addition, the disturbance attenuation issues for NCSs are investigated as well as stability problems. The strength of this approach comes from the solid theoretic results existing in the literature for stability, robust performance etc. for switched systems. By a switched system, we mean a hybrid dynamical system consisting of a finite number of subsystems described by differential or difference equations and a logical rule that orchestrates switching between these subsystems. Properties of this type of nodel have been studied for the past fifty years to consider engineering systems that contain relays and/or hysteresis. Recently, there has been a dramatic increase of interest in the stability analysis and switching control design of switched systems (see, for example, the survey papers $|12 \sim 15|$, recent books $[16,17]$ and the references cited thercin).

In this paper, we investigate the asymptotic stability and disturbance attenuation properties for a class of Networked Control Systems (NCSs) under uncertain access delay and packet dropout effects. Our aim is to tind conditions concerning the delay and packet dropout rate, under which the 
system stability and $\mathrm{H}_{\varnothing}$ disturbance attenuation properties are preserved to a desired level. We first analyze the nature of the uncertain access delay and packet dropout effects on NCSs in Section 2. Then in Section 3, we model the NCS as a discrete-time switched system. Therefore the NCSs' asymptotic stability and robust performance problems can be boiled down to the stability analysis and disturbance attenuation problems of switched systems. In Section 4, the asymptotic stability for such NCSs with uncertain access delay and packet dropout effects is studied, and disturbance attenuation properties for such NCSs are studied in Section 5. The techniques employed in this paper are based on recent progress in the continuous-time and discrete-time switched systems [18 20], i.e. multiple Lyapunov functions and average dwell time methods in particular. Finally, concluding remarks are presented.

\section{The access delay and packet dropout}

For the network link layer, we assume that the delays caused by processing and propagation are ignored. and we only consider the access delay which serves as the main source of delays in NCSs. Dependent on the data traffic, the communication bus is either busy or idle (available). If the link is available, the communication between sender and receiver is assumed to be instantaneous. Errors may occur during the communication and destroy the packet, and this is considered as a packet dropout.

The model of the NCS used in this paper is shown in Fig. 2. For simplicity, but without loss of generality, we may combine al] the time delay and packet dropout effects into the sensor to controller path and assume that the controller and the actuator communicate ideally.

We assume that the plant can be modeled as a continuoustime linear time-invariant system described by

$$
\left\{\begin{array}{l}
x(t)=A^{c} x(t)+B^{\prime \prime} u(t)+E^{t} d(t), \\
z(t)=C^{\prime \prime} x(t) .
\end{array}, \in \mathbb{R}^{+}\right.
$$

where $\mathbb{R}^{+}$stands for nonnegative real numbers, $x(t) \in \mathbb{R}^{n t}$ is the state variable, $u(t) \in \mathbb{R}^{n t}$ is control input, and $z(t) \in \mathbb{R}^{p}$ is the controlled output. The disturbance input $d(t)$ is contained in $D \subset \mathbb{R}^{r} . A^{*} \in \mathbb{R}^{n \times n}, B^{c} \in \mathbb{R}^{n \times m}$ and $E^{r} \in \mathbb{R}^{n \times r}$ are constant matrices related to the system state, and $C^{r} \in \mathbb{R}^{r \times n}$ is the output matrix.

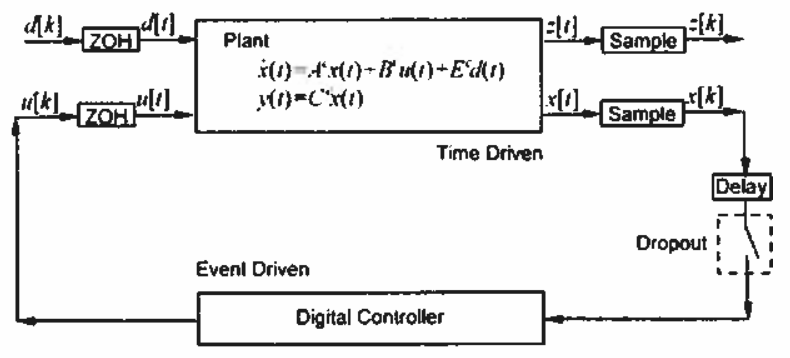

Fig. 2 The networked control systems' model.

For the above NCS, it is assumed that the plant output node (sensor) is time driven. In other words, after each clock cycle (sampling time $T_{\mathrm{s}}$ ), the output node attempts to send a packet containing the most recent state (output) samples. If the communication bus is idte, then the packet will be transmitted to the controller. Otherwise, if the bus is busy, then the output node will wait for some time, say $\varpi<T_{\text {s }}$, and try again. After several attempts or when newer sampled data become available, if the transmission still can not be completed, then the packet is discarded, which is also considered as a packet dropout. On the other hand, the controller and actuator are event driven and work in a simpler way. The controller, as a receiver, has a receiving buffer which contains the most recently received data packet from the sensors (the overflow of the buffer may be dealt with as packet dropouts). The controller reads the buffer periodically at a higher frequency than the sampling frequency. say every $T_{\mathrm{s}} / N$ for some integer $N$ large enough. Whenever there are new data in the buffer, the controller will calculate the new control signal and transmit it to the actuator. Upon the arrival of the new control signal, the actuator updates the output of the Zero-Order-Hold ( $\mathrm{ZOH}$ ) to the new value.

Based on the above assumptions, a typical time delay and packet dropout pattern is shown in Fig. 3. In this figure, the small bullet. - stands for the packet being transmitted successfully from the sensor to the controller's receiving buffer, 
maybe with some delay, and being read by the controller, at some time $t=k T_{s}+h\left(T_{s} / N\right)$ ( $k$ and $h$ are integers).

The new control signal is sent to the actuator and the actuator holds this new value until the next update control signal comes. The symbol. 0 , denotes the packet being dropped, due to error, bus being busy, conflict or buffer overflow etc.

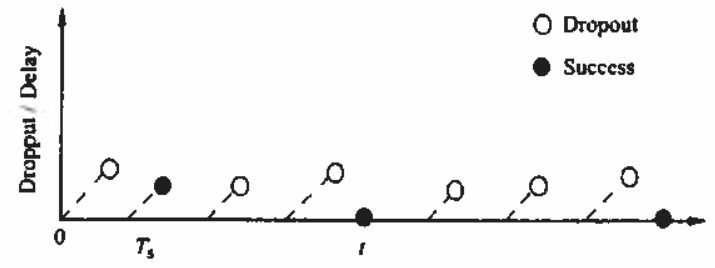

Fig. 3 The illustration of uncertain time delay and packet dropout of Networked Control Systems.

\section{Switched system models}

In this section, we will consider the sampled-data model of the plant. Because we do not assume the synchronization between the sampler and the digital controller, the control signal is no longer of constant value within a sampling period. Therefore the control signal within a sampling period has to be divided into subintervals corresponding to the controller's reading buffer period, $T=T_{\mathrm{s}} / N$. Within each subinterval, the control signal is constant under the assumptions of the previous section. Hence the continuous-time plant may be discretized into the following sampled-data systems:

$$
x[k+1]=A_{x}[k]+\left[B A_{T} B \cdots A_{\tau}^{N-1} B\right]\left[\begin{array}{c}
u^{1}[k] \\
u^{2}[k] \\
\vdots \\
u^{N}[k]
\end{array}\right]
$$$$
+E d[k]
$$

where $A=e^{A^{c} T_{*}}, A_{T}=e^{A^{r T}}, B=\int_{0}^{T} e^{A^{c} \eta} B^{c} \mathrm{~d} \eta$ and $E=\int_{0}^{T_{\kappa}} \mathrm{c}^{\prime \prime \prime} E^{\prime} \mathrm{d} \eta$. Note that for linear time-invariant plant and constant-periodic sampling, the matrices $A_{1} A_{T}$, $B$ and $E$ are constant. In addition, if the sampling period
$T_{s}$ is small enough and/or $N$ is large enough then one could approximate $A_{\tau}$ as an identity matrix and simplify the representation.

\subsection{Modeling uncertain access delay}

During each sampling period, there are several different cases that may arise.

First, if there is no delay, namely $\tau=0, u^{1}[k]=u^{2}[k]=$ $\cdots=u^{N}[k]=u[k]$, then the state transition equation (2) for this case can be written as:

$$
\begin{aligned}
x[k+1]= & A x[k]+\left[B A_{T} B \cdots A_{T}^{N-1} B\right]\left[\begin{array}{c}
u[k] \\
u[k] \\
\vdots \\
u[k]
\end{array}\right] \\
& +E d[k] \\
= & A x[k]+\sum_{i=0}^{N-1} A_{T}^{i} B u[k]+E d[k] .
\end{aligned}
$$

Secondly, if the delay $\tau=h \times T$, where $T=T_{\mathrm{s}} / N$, and $h=1,2, \cdots, d_{\max }{ }^{*}$, then $u^{1}[k]=u^{2}[k]=\cdots=u^{h}[k]=$ $u[k-1], u^{h+1}[k]=u^{h+2}[k]=\cdots=u^{N}[k]=u[k]$, and (2) can be written as:

$$
\begin{aligned}
x[k+1]= & A x[k]+\left[B A_{T} B \cdots A_{T}^{N-1} B\right]\left[\begin{array}{c}
u[k-1] \\
\vdots \\
u[k-1] \\
u[k] \\
\vdots \\
u[k]
\end{array}\right] \\
& +E d[k]) \\
= & A x[k]+\sum_{i=0}^{h-1} A_{T}^{i} B u[k-1]+\sum_{i=h}^{N-1} A_{T}^{i} B u[k] \\
& +E d[k] .
\end{aligned}
$$

Note that $h=0$ implies $\tau=0$, which corresponds to the previous "no delay" case.

Let us assume that the controller uses just the timeinvariant linear feedback control law, $u[k]=K x[k]$. which may be obtained as the solution of a LQR problem with-

\footnotetext{
- The vulue of $d_{\text {max }}$ is determined as the least integer greater than the positive scalar $\tau_{\text {twix }} / T$. where $\tau_{\text {max }} s$ tands for the maximum access delay,
} 
out considering the network induced effects. Then, we may we have

plug in the $u[k]=K x[k]$ and obtain

$$
\begin{aligned}
x[k+1]= & A x[k]+\sum_{i=0}^{h-1} A_{T}^{i} B K x[k-1] \\
& +\sum_{i=h_{k}}^{N-1} A_{T}^{i} B K x[k]+E d[k] \\
= & \left(A+\sum_{i=h}^{N-1} A_{T}^{i} B K\right) x[k] \\
& +\sum_{i=0}^{h-1} A_{T}^{i} B K x[k-1]+E d[k]
\end{aligned}
$$

If we let $\hat{x}[k]=\left[\begin{array}{c}x[k-1] \\ x[k]\end{array}\right]$, then the above equations can be written as:

$$
\begin{aligned}
\hat{x}[k+1]= & {\left[\begin{array}{cc}
0 & I \\
\sum_{i=0}^{h-1} A_{T}^{i} B K & A+\sum_{i=h}^{N-1} A_{T}^{i} B K
\end{array}\right] \hat{x}[k] } \\
& +\left[\begin{array}{c}
0 \\
E
\end{array}\right] d[k]
\end{aligned}
$$

where $h=1.2, \cdots, d_{\max }$. The controlled output $z[k]$ is given by

$$
z[k]=\left[\begin{array}{ll}
0 & C
\end{array}\right] \hat{x}[k]
$$

where $C=C^{e}$.

Finally, if a packet-dropout happens, which may be due to a corrupted packet or sending it out with delay greater than $\tau_{\text {max }}$, then the actuator will implement the previous control signal, i.e. $u^{1}[k]=u^{2}[k]=\cdots=u^{N}[k]=u[k-1]$. Therefore, the state transition equation (2) for this case can be written as:

$$
\begin{aligned}
x: k+\mathrm{l}]= & A x[k]+\left[B A_{T} B \cdots A_{T}^{N-1} B\right]\left[\begin{array}{c}
u[k-1] \\
u[k-1] \\
\vdots \\
u[k-1]
\end{array}\right] \\
& +E d[k] \\
= & A x[k]+\sum_{i=0}^{N-1} A_{T}^{i} B u[k-1]+E d[k] \\
= & A x[k]+\sum_{i=0}^{N-1} A_{T}^{i} B K x[k-1]+E d[k] .
\end{aligned}
$$

Using the same variable transformation as in the above case,

$$
\hat{x}[k+1]=\left[\begin{array}{cc}
0 & I \\
\sum_{i=0}^{N-1} A_{T}^{i} B K^{K} A
\end{array}\right] d[k]+\left[\begin{array}{l}
0 \\
E
\end{array}\right] d[k] .
$$

The controlled output $z[k]$ is given by

$$
z[k]=\left[\begin{array}{ll}
0 & C
\end{array}\right] \hat{x}[k] .
$$

where $C=C^{c}$.

\subsection{Switched system model}

In the following, we will model the uncertain multiple successive packet dropouts and formulate the above NCSs as a class of discrete-time switched systems.

Motivated by the above analysis of NCSs, we introduce a family of discrete-time linear systems described by the following difference equations.

$$
x[k+1)=A_{q} x[k]+E_{q} d[k], \quad k \in \mathbb{Z}^{+},
$$
where $x[k] \in \mathbb{R}^{n}$ is the state variable, and the disturbance input $d[k]$ is contained in $D \subset \mathbb{R}^{r} . A_{q} \in \mathbb{R}^{n \times n}$ and $E_{q} \in \mathbb{R}^{n \times r}$ are constant matrices indexed by $q \in Q$. where the finite set $Q=\left\{q_{1}, q_{2}, \cdots, q_{n}\right\}$ is called the set of modes.

Combine the family of discrete-time uncertain linear systems (3) with a class of piecewise constant functions of time $\sigma: \mathbb{Z}^{+} \rightarrow Q$. Then, we can define the following timevarying system as a discrete-time switched linear system

$$
x[k+1]=A_{\sigma[k]} x[k]+E_{\sigma[k]} d[k] ; \quad k \in \mathbb{Z}^{+} .
$$

The sequence $\sigma[k]$ is usually called a switching signal.

Associated with the switched system (4), a controlled output $z[k]$ is considered.

$$
z[k]=C_{\sigma(k)} x[k]
$$

where $C_{\pi[k]} \in \mathbb{R}^{n \times n}$ and $z[k] \in \mathbb{R}^{p}$.

For the NCS we considered in this paper, we may formu late it as a switched system with $d_{1 n \text { st } x}+2$ different modes, which can be expressed as follows.

$$
\left\{\begin{array}{l}
\hat{x}[k+1]=A_{h} \hat{x}[k]+E_{h} d[k] . \\
z[k]=C_{h} \hat{x}[k]
\end{array}\right.
$$


where $A_{h}=\left[\begin{array}{cc}0 & I \\ \sum_{h=0}^{h-1} A_{J}^{i} B K & A+\sum_{i=h}^{N-1} A_{r}^{i} B K\end{array}\right], E_{h}=$ $\left[\begin{array}{l}0 \\ E\end{array}\right]$ and $C_{h_{h}}=\left[\begin{array}{ll}0 & C\end{array}\right]$ for $h=0,1.2, \cdots d_{t \text { nix }}, N$. And the set of modes $Q$ is given by $Q=\left\{0,1,2, \cdots d_{\text {nax }} . N\right\}$. Note that $h=0$ implies $\tau=0$, which corresponds to the "no delay" case, i.e.

$$
A_{0}=\left[\begin{array}{cc}
0 & I \\
0 & A+\sum_{i=0}^{N-1} A_{T}^{i} B K
\end{array}\right]
$$

while $h=N$ corresponds to the "packet dropout" case.

In our previous work [21], it was assumed that there is an upper bound on the maximum number of successive packet dropouts, e.g., at most four packets dropped in a row. Under this assumption, NCSs under bounded uncertain access delay and packet dropout were modeled as switched linear systems with arbitrary switching. The asymptotic stability and persistent disturbance attenuation properties of the NCSs were studied in the switched system framework, and a necessary and sufficient condition was given for the NCSs' asymptotic stability in [21]. However, assuming an absoiute upper bound on the maximum number of packets dropped in a row could be conservative in certain cases. In this paper, we provided an alternative way to model NCSs as switched systems. Instead of incorporating all possible delay-dropout patterns so as to relax the switching signal to be arbitrary, we specified a subclass of the switching signal by restricting the occursing frequency and the number of dropped and seriously-delayed packets in the time average sense. In particular, it was shown that there exist bounds on the delay and packet dropout rate and percentage, below which the NCSs" stabjlity and $L_{2}$ disturbance attenuation properties may be preserved to a desired level. These bounds were identified based on multiple Lyapunov functions incorporated with average dwell time scheme.

\section{Stability analysis}

Considering the switched system model for NCSs (5). it is reasonable to assume that. for the cases of no delay $(h=0)$ or smatl delay $\left(h \leqslant h_{1}\right)$, the corresponding state matrix $A_{h}$ 's are Schur stable, while, for the cases of large delay $\left(h>h_{0}\right)$ or packet dropout $(h=N)$, the $A_{h}$ 's are not Schur stable. Therefore, in this paper it is assumed that the first $r$, corresponding to $h_{(0)}$, of all the $d_{\text {mat }}+2$ matrices in $\left\{A_{J_{r}}\right\}$ are Schur stable. while the rest matrices are not Schur stable, where $r \leqslant d_{\text {max }}+2$ and $h \in$ $Q=\left\{0,1,2, \cdots, d_{\max }, N\right\}$. In the sequel, for simplicity of notation, we will index the switched NCS model with $i$, for $i \in Q=\left\{0.1 .2, \cdots . d_{\operatorname{sm}} . N\right\}$, and replace $\hat{x}$ as $x$. In this section, we set $d[k]=0$ in (5) for the purpose to study its stability.

It is known that for Schur stable systems $x[k+1]=$ $A_{i} x[k]$, there always exist positive scalars $\lambda_{1}<\mathrm{I}$ and $h_{i}$ 's, $i \leqslant r$ such that $\left\|A_{i}^{k}\right\| \leqslant h_{i} \lambda_{1}^{k}$ for any $k \geqslant 1^{* *}$. Note that for any Schur unstable system $x[k+1]=A_{i} x[k](i>r)$, there always exist a constant $0<\sigma<\mathrm{l}$ making the system $x[k+1]=\sigma A_{k} x[k]$ Schur stable. Hence we may assume that there exist positive scalars $\lambda_{2} \geqslant 1$ and $h_{i}$ 's, $i>r$ such that $\left\|A_{i}^{k}\right\| \leqslant h_{1} \lambda_{2}^{k}$ for any $k \geqslant 1$. Therefore, we obtain

$$
\left\|A_{i}^{k}\right\| \leqslant \begin{cases}h_{i} \lambda_{1}^{k}, & i \leqslant r \\ h_{i} \lambda_{2}^{k}, & i>r\end{cases}
$$

Following [20], we introduce the notations as below. Denote $h=\max _{i}\left\{h_{i}\right\}$. For any switching signal $\sigma(k)$ and any $k_{2}>k_{1}>0$, let $N_{\sigma}\left(k_{1}, k_{2}\right)$ denote the number of switchings of $\sigma(k)$ on the interval $\left[k_{1}, k_{2}\right)$. Let $K_{i}\left(k_{1}, k_{2}\right)$ denote the total period that the $i$-th subsystem is activaled during $\left[k_{1}, k_{2}\right)$. Define

$$
K^{-}\left(k_{1} \cdot h_{2}\right)=\sum_{i \leqslant n \in Q} K_{i}\left(h_{1} \cdot k_{2}\right) .
$$

which stands for the total activation period of the Schur stable subsystems. On the other hand.

$$
K^{+}\left(k_{1}, k_{2}\right)=\sum_{i, r_{i} \in Q} K_{i}\left(k_{1}, k_{2}\right)
$$

denotes the total activation period of the Schur unstable subsystems. We have $K^{-}\left(k_{1}, k_{2}\right)+H^{+}+\left(k_{1}, k_{2}\right)=k_{2}-k_{1}$.

For given $N_{0} \geqslant 0, \tau_{a}$, let $S_{a}\left(\tau_{a}\right)$ denote the set of all switching signals satisfying

$$
N_{\sigma}(0, h) \leqslant N_{0}+\frac{k}{\tau_{r}}
$$

\footnotetext{
". The matrix norm is defined as the induçed matrix norm from vector 2-norn, i.c. $\|x\|=\sqrt{\sum_{i=1}^{n} n_{i}^{2}}$.
} 
where the constant $T_{i \mathrm{i}}$ is called the average dwell time and $N_{i j}$ the chatter bound. The idea is that there may exist consecutive switching separated by less than $\tau_{\mathrm{n}}$, but the average time interval between consecutive switchings is not less than $\tau_{\mathrm{a}}$. Note that the concept of average dwell time between subsystems was originally proposed for continuoustime switched systems in [22]. With these assumptions and notations, we may apply the techniques and results developed in [20] to the NCSs and get the following theorem for globally exponential stability. The proof of the theorem is not difficult by using the technique of Theorem 3 in [20], and thus is omitted here.

Theorem 1 For any given $\lambda \in\left(\lambda_{1}, 1\right)$, the NCS (5) is globally exponentially stable with stability degree $\lambda$ if there exists a finite constant $r_{\mathrm{a}}^{*}$ and $\lambda^{*} \in\left(\lambda_{1}, \lambda\right)$ such that the $K^{+}(0, k)$ and $N_{\sigma}(0, k)$ satisfy the following two conditions:

$$
\text { 1) } \quad \inf _{k \rightarrow 0} \frac{K^{-}(0, k)}{K^{*}(0, k)} \geqslant \frac{\ln \lambda_{2}-\ln \lambda^{*}}{\ln \lambda^{*}-\ln \lambda_{1}}
$$

holds for some scalar $\lambda^{*} \in\left(\lambda_{1}, \lambda\right)$;

2) The average dwell time is not smaller than $\tau_{i}^{*}$, i.e.

$$
N_{\sigma}(0, k) \leqslant N_{0}+\frac{k}{\tau_{i t}^{*}},
$$

where

$$
\tau_{a}^{*}=\frac{\ln h}{\ln \lambda-\ln \lambda^{*}},
$$

and $N_{0}$ may be specified arbitrarily.

Remark 1 The first condition implies that if we expect the entire system to have decay rate $\lambda$, we should restrict the total number of lost packets and large delay packets in the sense that on average $K^{+}(0, k)$ has an upper-bound,

$$
K^{+}(0, k) \leqslant \frac{\ln \lambda^{*}-\ln \lambda_{1}}{\ln \lambda_{2}-\ln \lambda_{k}} k .
$$

That means the percentage of dropped and seriously delayed packets should be below certain bound, given by

$$
\frac{\ln \lambda^{*}-\ln \lambda_{1}}{\ln \lambda_{2}-\ln \lambda_{1}}
$$

Remark 2 The main point of the second condition can be described as follows. Although the first condition may be satisfied. which means that on average the packet lost is limited and the total number of large delayed packet is bounded, in the worst case the packet dropout and large access delay happen in a burst fashion. For such worst case. the NCSs may fail to achieve the decay rate. The second condition restricts the frequency of the packet dropout and large delayed packet, and to make sure the above worst calse can not happen.

Remark 3 The above theorem says that the NCSs' stability. with most of the packets arriving in a timely fashion, does not degenerate seriously, which is reasonable.

\section{Disturbance attenuation properties}

In this section, we will study the disturbance attenuation property for the NCSs (5). Note that the $L_{2}$ gain property of discrete-time switched systems was studied in [20] under the assumption that all subsystems were Schur stable. In this section, we will extend the $L_{2}$ gain property of discretelime switched system to the case that not all subsystems are Schur stable. The techniques used in this section are similar to those in [19] for continuous-time switched systems.

Following the assumptions in [20], the initial state is assumed to be the origin, $x[0]=0$. And we assume that the Schur stable subsystems achieve an $L_{2}$ gain smaller than $\gamma_{0}$. It is known that there exist a positive scalar $\lambda_{-}<1$ and a set of positive definite matrices $P_{i}$, for $i \leqslant r$ and $i \in Q$, such that

$$
\begin{aligned}
& A_{i}^{\mathrm{T}} P_{i} A_{i}-\lambda_{-}^{2} P_{i}+C_{i}^{\mathrm{C}} C_{i} \\
& +A_{i}^{\mathrm{T}} P_{i} E_{i}\left(\gamma_{0}^{2} l-E_{i}^{\mathrm{T}} P_{i} E_{i}\right)^{-1} E_{i}^{\mathrm{T}} P_{i} A_{i}
\end{aligned}
$$

is negative definite [23]. Observing that for Schur unstable subsystems, there always exist a constant $0<\sigma<1$, such that the subsystems $\left(\sigma A_{i}, E_{i}, \sigma C_{i}\right)$ can achieve the $L_{2}$ gain level $\gamma_{0}$. Therefore, we assume that for Schur unstable subsystems there exist a positive scalar $\lambda_{+} \geqslant 1$ and a set of positive definite matrices $P_{i}$, for $i>r$ and $i \in Q$, such that

$$
\begin{aligned}
& A_{i}^{\mathrm{T}} P_{i} A_{i}-\lambda_{+}^{2} P_{i}+C_{i}^{\mathrm{T}} C_{i} \\
& +A_{i}^{\mathrm{T}} P_{i} E_{i}\left(\gamma_{i 0}^{2} I-E_{i}^{\mathrm{T}} P_{i} E_{i}\right)^{-1} E_{i}^{\mathrm{T}} P_{i} A_{i}
\end{aligned}
$$

is negative definite. Using the solution $P$, "s, we define the following piecewise Lyapunov function candidate

$$
V(k)=V_{\sigma|k|}(x)=x^{i}[k] P_{\sigma|k| x}[k]
$$

for the switched system, where $I_{\sigma(k)}$ is switched among the solution $P_{i}$ 's in accordance with the piecewise constant switching signal $\sigma[k]$. It can be shown as in $[20]$ that there always exist constant scalars $\alpha_{1}, \alpha_{2}>0$, for example, 
$\alpha_{1}=\inf _{i \in Q} \lambda_{m}\left(P_{i}\right), \alpha_{2}=\sup _{i \in Q} \lambda_{M}\left(P_{i}\right)$, such that

$$
{ }^{\prime} x_{1}\|x\|^{2} \leqslant V_{i}(x) \leqslant a_{2}\|x\|^{2} . \forall \cdot x \in \mathbb{R}^{n}, \forall i \in Q .
$$

Here $\lambda_{w}\left(P_{i}\right)$ and $\lambda_{m}\left(P_{t}\right)$ denotes the largest and smallest eigenvalue of $P_{i}$ respectively. There exist a constant scalar $\mu \geqslant 1$ such that

$$
V_{i}(x) \leqslant \mu V_{j}(x), \forall x \in \mathbb{R}^{n}, \forall i, j \in Q
$$

A conservative choice is $\mu=\operatorname{sip}_{k, V \in Q} \frac{\lambda_{M}\left(P_{k}\right)}{\lambda_{m}\left(P_{l}\right)}$.

Following the steps in [20]. for each $V_{i}(x)=$ $x^{\mathrm{T}}[k] P_{i} x[k]$ along the solutions of the corresponding subsystem, we may obtain that

$$
\begin{aligned}
& V_{i}(x[k+1])-V_{i}(x[k]) \\
& \leqslant\left\{\begin{array}{l}
-\left(1-\lambda_{-}^{2}\right) V_{i}(x[k])-z^{\mathrm{T}}[k] z[k]+\gamma_{0} d^{\mathrm{T}}[k] d[k] . \\
-\left(1-\lambda_{+}^{2}\right) V_{i}(x[k])-z^{\mathrm{T}}[k] z[k]+\gamma_{0} d^{\mathrm{T}}[k] d[k] .
\end{array}\right.
\end{aligned}
$$

For a piecewise constant switching signal $\sigma[k]$ and any given integer $k>0$, we let $k_{1}<\cdots<k_{i}(i \geqslant 1)$ denote the switching points of $\sigma[k]$ over the interval $[0, k)$. Then, using the above difference inequalities, we obtain

$$
V(k) \leqslant\left\{\begin{array}{l}
\lambda_{-}^{2\left(k-k_{i}\right)} V\left(k_{i}\right)-\sum_{j=k_{i}}^{k-1} \lambda_{-}^{2(k-1-j)} \Gamma(j), \\
\lambda_{+}^{2\left(k-k_{i}\right)} V\left(k_{i}\right)-\sum_{j=k_{i}}^{k-1} \lambda_{+}^{2(k-1-j)} \Gamma(j),
\end{array}\right.
$$

where $\Gamma(j)=z^{\mathrm{T}}[j] z[j]-\gamma_{0}^{2} d^{\mathrm{T}}[j] d[j]$. Since $V\left(k_{i}\right) \leqslant$ $\mu V\left(k_{i}^{-}\right)$holds on every switching point $k_{i}$, we obtain by induction that

$$
\begin{aligned}
V(k) \leqslant & \mu^{N_{\sigma}(0, k)} \lambda_{-}^{2 K^{-}(0, k)} \lambda_{+}^{2 K^{+}(0, k)} V(0) \\
& -\sum_{j=0}^{k-1} \mu^{N_{\sigma}(j \cdot k-1)} \lambda_{-}^{2 K^{-}(j, k-1)} \lambda_{+}^{2 K^{+}(j \cdot k-1)} \Gamma(j) \\
= & -\sum_{j=0}^{k-1} \mu^{N_{-}(j \cdot k-1)} \lambda_{-}^{2 K^{-}(j, k-1)} \lambda_{+}^{2 K^{+}(j, k-1)} \Gamma(j) .
\end{aligned}
$$

The last equality is because of the zero initial state assumption $x[0]=0$.

We assume that on any interval $\left[k_{1}, k_{2}\right)$ the total activation periods of the unstable subsystems satisfies $K^{+}\left(k_{1}, k_{2}\right) \leqslant \frac{\ln \lambda^{*}-\ln \lambda_{-}}{\ln \lambda_{+}-\ln \lambda_{-}}\left(k_{2}-k_{1}\right)$, or equivalently

$$
\frac{K^{-}\left(k_{1} \cdot k_{2}\right)}{K^{+}\left(k_{1} \cdot k_{2}\right)} \geqslant \frac{\ln \lambda_{+}-\ln \lambda^{*}}{\ln \lambda^{*}-\ln \lambda_{-}}
$$

holds for some scalar $\lambda^{*} \in\left(\lambda_{-}, 1\right)$ and $\forall k_{2}>k_{1} \geqslant 0$. Then, we have

$$
\begin{aligned}
& \lambda_{-}^{2 K^{-}(j, k-1)} \lambda_{+}^{2 k^{*}(j, k-1)} \\
& \leqslant\left(\lambda^{*}\right)^{2 K^{-}(j, k-1)+2 k^{*}(j \cdot k-1)}=\left(\lambda^{*}\right)^{2(k-1-j) .}
\end{aligned}
$$

Therefore, we obtain

$$
V(k) \leqslant-\sum_{j=0}^{k-1} \mu^{N_{n}(j \cdot k-1)}\left(\lambda^{*}\right)^{2(k-1-j)} \Gamma(j) .
$$

When $\mu=1$, we have from $V(k) \geqslant 0$ and (12) that

$$
\sum_{j=0}^{k-1} \mu^{N_{0}(j-k-1)}\left(\lambda^{*}\right)^{2(k-1-j)} \Gamma(j) \leqslant 0 .
$$

We sum (13) from $k=1$ to $k=+\infty$ to obtain

$$
\begin{aligned}
& \sum_{k=1}^{+\infty}\left(\sum_{j=0}^{k-1} \mu^{N_{m}(j \cdot k-1)}\left(\lambda^{*}\right)^{2(k-1-j)} \Gamma(j)\right) \\
& =\sum_{j=1}^{+\infty} \Gamma(j)\left(\sum_{k=j+1}^{+\infty} \mu^{N_{o}(j, k-1)}\left(\lambda^{*}\right)^{2(k-1-j)}\right) \\
& =\left(1-\left(\lambda^{*}\right)^{2}\right)^{-1} \sum_{j=1}^{+\infty} \Gamma(j) \leqslant 0 .
\end{aligned}
$$

which means

$$
\sum_{j=0}^{+\infty} z^{\mathrm{T}}[j] z[j] \leqslant \gamma_{0}^{2} \sum_{j=0}^{+\infty} d^{\mathrm{T}}[j] d[j] .
$$

Therefore, $L_{2}$ gain $\gamma_{0}$ is achieved for the switched system, namely the NCS (5).

For the case $\mu>1$, multiplying both sides of (12) by $\mu^{-N_{\sigma}(0 . k-1)}$ leads to

$$
\begin{aligned}
& \sum_{j=0}^{k-1} \mu^{-N_{\sigma}(0, j)}\left(\lambda^{*}\right)^{2(k-1-j)} z^{\mathrm{T}}[j] z[j] \\
& \leqslant \gamma_{0}^{2} \sum_{j=0}^{k-1} \mu^{-N_{\sigma}(0 . j)}\left(\lambda^{*}\right)^{2(k-1-j)} d^{\mathrm{T}}[j] d[j] .
\end{aligned}
$$

Now, we choose a positive scalar $\lambda$ larger than 1 to consider the following average dwell time condition: for any positive integer $j>0$,

$$
N_{\sigma}(0, j) \leqslant \frac{j}{\tau_{\mathrm{at}}^{*}}, \quad \tau_{\mathrm{n}}^{*}=\frac{\ln \mu}{2 \ln \lambda} .
$$

Therefore $\mu^{-N_{n}(0, j)}>\lambda^{-2 j}$ holds for any $j>0$, where $\lambda=\mu^{\left(2 \tau_{\mathrm{in}}^{*}\right)^{-1}}$. Then. from (15) we obtain

$$
\begin{aligned}
& \sum_{j=0}^{k-1} \lambda^{-2 j}\left(\lambda^{*}\right)^{2(k-1-j)} z^{\mathrm{T}}[j] z[j] \\
& \leqslant \gamma_{n}^{2} \sum_{j=0}^{k-1}\left(\lambda^{*}\right)^{2(k-1-j)} d^{\mathrm{T}}[j] d[j] .
\end{aligned}
$$

Similarly, summing both sides of the above inequality from $k=1$ to $k=+\infty$ leads to

$$
\begin{aligned}
& \left(1-\left(\lambda^{*}\right)^{2}\right)^{-1} \sum_{j=1}^{+\infty} \lambda^{-2 j} z^{\top}[j] z[j] \\
& \leqslant\left(1-\left(\lambda^{*}\right)^{2}\right)^{-1} \gamma_{i j}^{2} \sum_{j=1}^{+\infty} d^{[}[j] d[j]
\end{aligned}
$$

and thus

$$
\sum_{j=1}^{+\infty} \lambda^{-2 j} z[j] z[j] \leqslant \gamma_{0}^{2} \sum_{j=1}^{+\infty} d^{\mathrm{T}}[j] d[j]
$$


holds for any $d[k] \in L_{2}[0 .+\infty)$. Following the notation in [20], we say that a weighted $L$. gain on is achieved. In summary, we prove the following theorem.

Theorem 2 The NCS (5) achieves a weighted $L_{2}$ gain $\gamma_{0}$ if the $K^{+}\left(k_{1}, k_{2}\right)$ satisfies $(1 \mathrm{I})$ and $N_{\sigma}(0, k)$ satisfy the condition of (16).

Remark 4 Similarly, the condition (11) restricts the number of packets that have been dropped out or delayed excessively, while the condition (16) restricts the happening frequency of them. Both of the conditions are given in the sense of average over time.

\section{Conclusions}

In this paper, we modeled a class of NCSs under uncertain access delay and packet dropout as discrete-time switched linear systems. The stability and disturbance attenuation issues for such NCSs were studied in the framework of switched systems. It was shown that the asymptotic stability and disturbance attenuation level might be preserved for the NCSs tunder certain bounds on the umount and rate of the dropped and large delayed packets. Although we only consider state feedback control laws here, the techniques and results developed here can be casily extended to the case of static output feedback control laws. It should be pointed out that the conditions concerning the delay and packet dropout rate for the preservation of the NCSs' stability and $\mathrm{H}_{x}$ disturbance attenuation properties were based on Lyapunov theory. Therefore, the conditions are sufficient only and maybe conservative for some cases.

We believe that switched system approaches to NCSs: are pronising, as many rescarch topics like networked continuous-controller design, controller and scheduling policy co-design could be pursued in the switched system framework. For example, in $\left\{24 \mid\right.$, a stability and $L_{2}$ performance preserving network bandwidth mamalgement policy was proposed based on switched systems approaches. The potential of dealing with NCSs as switched systems comes from the existence of solid theoretic results in the ficld of switched systems. jump linear systens etc. Interested readers may refer $10|15,25|$ for surveys on the most recent progress on switched linear systems.

\section{References}

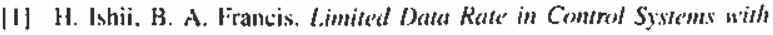

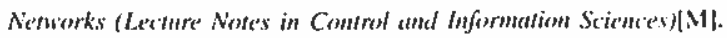
Berlin: Springer-Verlig. 2002. 275.

[2] W. Zhang. M. S. Branicky. S. M. Philliph. Stability of networked comtrol systems[J]. IEEE Contrul Systems Maga:me. 2001, 21(1):8449.

[3] R. W. Brokktu. D. I.jherzon. Quantized lecdback slubilizalion of

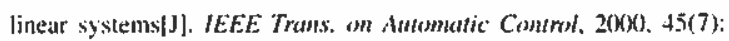
1279-1289.

Ifl W. S. Wong. R. W. Brockell. Systems with finite communication bandwidth consurants $I$ : stabilization with limited information [eedback|J!. IEEE Trans. on Aluomatic Comerol, 1999. 44(5): 10491053.

[5] N. Ejia, S. K. Mitcer. Stabilization of linear systems with limited

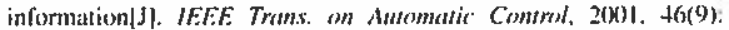
1384-1400

|6| P. Antsuk lis. J. Biallicul. Guest editorial: Special issue on networked control systenis[J]. IEEE Trans. on dufomatic Control. 2004. 49(9): $1241-1243$

$17 \mid$ D. F. Delchamps. Stabilizing a linear system with quantixed statc feedback[J]. IEEE Trans. on Aunomatic Commol. 1990, 35(8): 916924

[8] S. Tatikonda. S. Mitter, Control under communication contstraints|J]. IEEE frans. fan Autenustic Control. 2004, 49(7): 1056-1068.

[9] G. Natr. R. Lvans. I. M. Y. Mallects. W. Moran. Topological feedback entropy and nonlinear stabjlizalion:J. IEEE Troms. on Autonndic Comirol. 2004. 49(9): 1.585-1597.

[10] M. S. Branicky, S. M. Plijllips, W. Zhang. Stability of networked controt systems: explicit analysis of delay $|\mathrm{C}| /$ Proc: of $20 \% 0$ dinerican Contol Comf. Danvers. Masssachusetts: American Autormatic Control Council. 2000: 2352-2357.

[1] | A. Hassibi. S. P. Boyd. J. P. How. Control ol asynchronous dynamical systems with rate constraints on events|C|//Proc of the 38 ih IEEE Confo m Decisien and Commod. Piscaltawaly, New Jency: ItFE Press. [909: 1345-13.3.

112| D. l.iberron. A. S. Morse. Basic problems in stability and design if switched systems|Jl. IFEE Comm Sistems Magaine. 1999. 19(5): \$9-70.

[13] A. A. Niched. Recent trends in the stability anillysis of hybrid

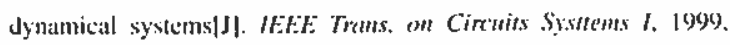
f6(1): 120-1.34.

[14] R. A. Decarlo. M. S. Branicky. S. Petterwon. B. Lemaarison. Perspectives and resutts an the stability and stabilizability of' bybrid

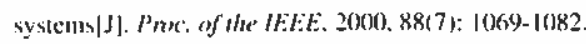

|IS| Z. Sun. S. S. Gc. Antilysis and byohesis of switched linear control systems|J]. Awesumeticta. 2005, +1(2): 181-195.

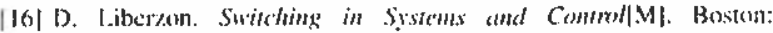
Hirklidustr. 2003 
117] Z. Sun, S. S. Ge. Suilched Linear Systems: Commol and Design|M|. New York: Springer-Verlig. 2005.

118| J. P. Hespanha. Liniform slahility of switched linear systems: Fxtensions of Labsulle's invariance principle[(J]. IEEE Trans. am Ausomatic Comtrol, $2004+49(4)$; 470-482.

|19| G. Zhai. B. Hu. K. Yusuda, A. N. Michel. Disturbance antenuation properties of time-controlled switched systens|J|. J. of the Franklin Instiume, 2001. 338(7): 765-779.

\{20| G. Zhai, B. Hu, K. Yasuda. A, N. Michel. Qualitative analysis of discrele-time switched systems $\mid C]$ /PFro. of 2002 Americin Comemol Comf.. Danvers, Massalchusetts: Americin Automatic Control Councit. 2002: 1880-1885.

[21] H. Lin, P. J. Antsaklis. Persistent disturbance atlenuation properties for net worked control systems [C]// Proce of the tird IFEE Conf. on Decision and Control. Piscalawaly, New Jercy: IEEE Pruss. 2004: 953958.

[22] J. P. Hespunha, A. S. Morse. Stability of Switched Systems with Average Dwell-time [C] //Proc: of the 38th IEEE Conf. on Decirion and Conurol. Piscalarvay, New Jercy: IEEE Press. 1999: 2655-2660.

[23] P. Gahinet, P. Apkarian. A linear matrix inequality approach to $H_{>0}$ control[]]. Int. J. of Robust and Nonlinear Control. 1994, 4(4): 421448.

[24] Fi. Lin, G. Zhai, 1. Fang, P. J. Antsaklis. Stability and $H_{\infty}$ performance preserving scheduling policy for networked control systerns|C| // Proce of the IGih IFAC World Congress on Alutomatic Comrol. Prague. Crech: Elsevier Science, 2005.

[25] H. Lin and P. J. Antsaklis. Stability, stabilizability of switched linear systems: a short survey of recent resultsfCI // IEFE Infernasionat Symposium on Intelligen Control and 13th Mediterranean Conf. on Comprol and Auromarion. Piscataway, New Jercy: IEEE Press. 2005: 24-30.

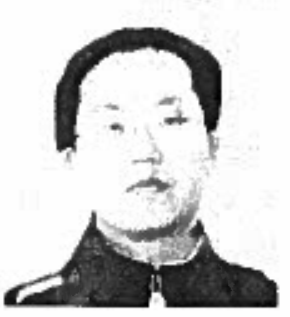

Hai LIN was born in Hebei, China, in 1976. He received the B.S. degree from University of Science and Techmology. Beijing. China in 1997, the M.E. degree from Chinese Academy of Science. China in 2000, and the Ph.D. degree from the University of Niotre Dame, USA in 2005. He is currently a post-dochoral research assuciate in the

EF Departmett at the University of Notre Dante. and is going to join the National biniversity of Singajore as an ansistiant professor in June zolo6. His research jnterests are in the multidistiplinary study of the problems at the intersection of conteol, commumiteation, computalion and life sciences. He is particularly incersited in hybrid systems theory. metworked controt. ind systems hiology.

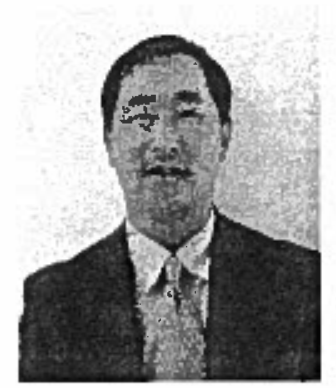

Guisheng 2HAl was borm in Hubei, Chinis. in 1967. He received the B.S. degree from Fudan Unitersity. China, in 1988 , and received the M.F. and the Ph.D. degrees. buth in system science. from Kobe Universily. Japan. in 1993 and 19y6, respectivaly. From 1996 to 1998. he worked in Kansai Laboratory of OKL Electric Industry Co. Lto.. Japan. From 1998 to 2004 , he was a research associate in the Departinent of Opto-Mechatronics, Wakayama University. Japan. He held a visiting research possition in the Department of Electrical Fngineering. the University of Notre Dane, USA. from August 2001 to July 2002. In April 2004, he joined the faculty of Osaka Prefecture Universily, Jipan, where he currently is an associate proliessor of Mechanical Engineering. His research interests include large scale and decentralized control systems, robust conIrol, switched/hybrid systems and switching control, networked control systems, neural networks and signal processing, ete. He is a senior member of IEEE, a member of ISCIE, SICE and JSME.

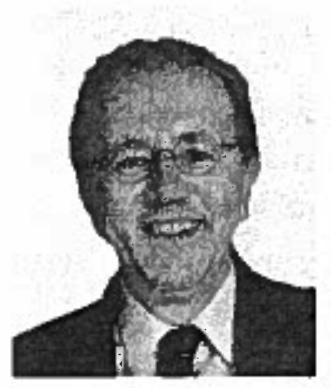

Panos J.ANTSAKI.IS reseived the undergratuale degree from the Nalional Technical Universily of Athens (NTUA), Alhens, Greece. and the M.S. and Ph.D. degrees fiom Brown University, USA. He is the H.C. and E.A. Brosicy Prolessor of Elcetrical Enginecring at the University of Notre Dame. USA. His work includes analysis of behavior and desigh of control stralegics for complex autonomous and intelligent systems. Flis recent reseurch focuses on networked embedded systems and addresses problems in the interdisciplinary resentch area of control. computing and communication networks. and on hybrid and discrete-event dynamical systems. He hats authored a number of publicalouns in journals.conference procecdings. and books. and he has edited several books on intelligent aulonomous comtrol and on hybrid systems. In addition, he has coatulhored the rescurch monograph Supervisory Con[rol of Diserete Event Systems Using Petri Nats INorwell, MA: Ktuwer. 1998) and the graduate textbeok I.inear Systems (New York: MeGrawHill. 1997). Dr. Antsaklis serves an the editurial buards of several jerurads. He has served as Brogram Chair and Geleral Chair of mijor systems and control conferencess. ils a Momber and Chair of comunteses in the IEEE. and IFAC, and he was the 1997 President of the IFI:F Control Systerns Society (CSS). He is is Distinguishad Ieeturer of the IElEE Control Systems Socicty. a recipient of the JEEE Distinguished Member Awatel of the Control Systens Society. and an LEEE. Third Millennium Madal recipient. 